\title{
Ranger, an Example of Integration of Robotics into the Home Ecosystem
}

\author{
Francesco Mondada ${ }^{1}$, Julia Fink ${ }^{2}$, Séverin Lemaignan ${ }^{2}$, David Mansolino ${ }^{3}$, \\ Florian Wille ${ }^{4}$, and Karmen Franinović ${ }^{4}$ \\ 1 Laboratoire de systèmes robotiques (LSRO) \\ 2 Computer-Human Interaction in Learning and Instruction Laboratory (CHILI) \\ 3 Distributed Intelligent Systems and Algorithms Laboratory (DISAL) \\ Ecole Polytechnique Fédérale de Lausanne (EPFL), Switzerland \\ 4 Zürcher Hochschule der Künste (ZHdK), Zürich, Switzerland
}

\begin{abstract}
This paper presents the concept and an example of robject, a robotic entity embedded in an everyday object. Robjects use the affordance of the original object to ensure an efficient interaction and a high acceptance. The example of the ranger robot shows that this approach can be applied to the domestic environment. We explore the integration of a robot (robject) into a family household, by regarding the home as a ecosystem, which consists of people, parts, products, activities, and interactions. A test of the ranger robot in families validates this holistic approach and shows the impact of this type of design in respect to the complexity of the robotic system.
\end{abstract}

Keywords: holistic approach, ecosystem, cooperation with humans, domestic service robots, robject, ranger robot

\section{Introduction}

Since years, predictions say service robotics will massively enter in every home [1]. In Europe, a large survey made in 2012 shows a general public perception which is still not very open to home service robotics. Robots are perceived as a good tool mainly for dangerous tasks [2]. In the same survey, a majority of people thinks robots should be banned from typical home service scenarios that include children, elderly or disabled care. Only $13 \%$ of the European citizen think robots should be applied in priority to "domestic use, such as cleaning". Also among researchers it has not been clear what a robot exactly should do in homes [3]. Pantofaru et al. [3] explored the role of robots in home organization (tidying, storing), and found that robots could have a potentially high impact on this. Similarly, Bell et al. [4] suggest that robots could be used in tidying up scenarios in the domestic environment.

Although only few such systems have entered the consumer market, several researchers took advantage of these few success and studied the acceptance of robotics technology by the users. Bauwens et al. [5] showed that the main factors impacting the adoption of robotics at home are, from the most to the less important: 
1. The practical utility

2. The integration into the home ecosystem (physical space, users, habits)

3. The economic utility

Most of the service robots developed in research only look at a subset of these criteria, approaching single disciplines such as HRI, mechatronics or robotic functionality.

In this paper we present an holistic approach and an example of mechatronic implementation looking for a balance between functionality, cost and integration into the ecosystem. We believe that an holistic and interdisciplinary approach can improve acceptance and bring robotics in homes in a faster and more efficient way. Our approach consists in integrating robotics technology into daily objects, making them what we call robjects. Robjects can easily blend into the home ecosystem because of the embodiment in an object that is already integrated into the ecosystem and has a clear function in it. Robjects also aim at a close synergy with the users, replacing high technological requirements with better human-robot interaction for collaboration. These principles have already been applied to some successful systems in industry, as illustrated in section 2. Section 3 presents an example of robject and the first results of user tests.

\section{State of the art}

In homes, research aims at adding intelligence into home automation, for instance to implement user activity recognition [6] and integrate service robots [7]. In most cases this technology, which is still very heavy, targets assistive living for elderly. A set of European research projects work exactly on this issue: Robot-ERA[8] is developing several service robots for indoor and outdoor use, integrated in smart houses and providing a set of services for older people, Giraffe $+[9]$ integrates a telepresence robot into a smart home where the sensor network allows context recognition and interpretation of collected data, ACCOMPANY[10] develops a robotic companion integrated into an intelligent environment, providing services to elderly users to enable independent living at home, MOBISERV[11] aims at studying innovative technology to support independent living of older adults at home or in specialized institutions, CompanionAble[12] has worked on the synergy between robotics and ambient Intelligence technologies enabling a care-giver's assistive environment and HOBBIT[13] develops a socially assistive robot that helps elderly at home. All these projects involve robots that have a human-like presence in the environment. Few projects such as RUBICON[14] have a more general approach and study a more generic network of sensors and actuators, without a predefined aapplication of assisted living in mind. Finally only very few consider the interaction with children. The MOnarCH project [15] is studying the interaction with children in hospitals with a smaller robot, still having a human-like shape. Their study on interaction is probably one of the closest to our project.

The integration of designers in research projects to study interaction aspects and gather information on new approaches is very recent in the field. A good 


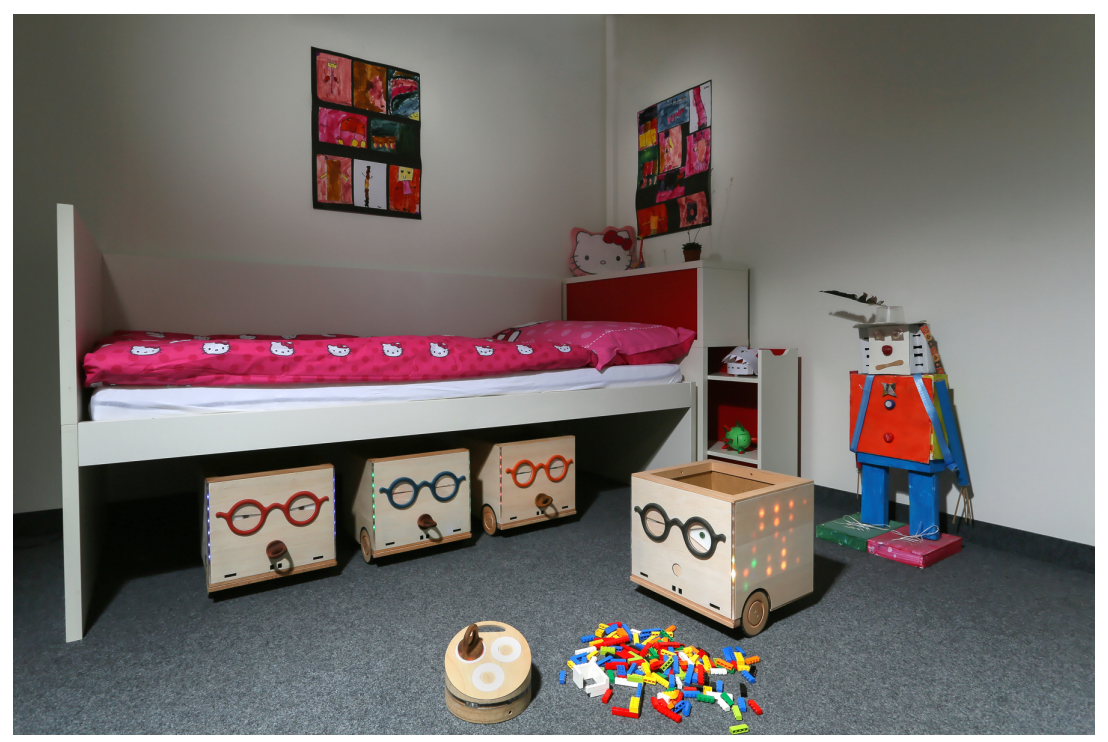

Fig. 1: A set of ranger robots in a room. Their home is under the bed, where they sleep recharging their battery. Photo: Alain Herzog.

experiment has been carried out in the University of Hertfordshire Robot House, where artists lived full-time with various robots in a smart home environment [16]. This approach gives many insights on interactions aspects, as we saw in our project.

Although most of the service robots developed in research lab do not meet market requirements or address only part of the requirements, few managed to become successful products. Among them we can find the Kiva systems [17] or the Baxter robot [18], both systems focusing on cooperation with humans, functional shapes, low cost and high added value functionalities. This situation confirms what Takayama et al. [19] found: "people would feel more positively toward robots doing occupations with people rather than in place of people". Transposing this approach to homes for a use with children, we developed a robot to help tidying-up the children's room, which has been considered as an interesting task by previous studies $[3,4]$.

\section{The ranger robot}

The ranger robot (see figure 1) is based on a common object that can be found in every children room: a wooden storage box for toys. This object has been transformed into what we call a robject, i.e. an object augmented with robotic features. This transformation has beed carried out by an interdisciplinary team including mechatronic engineers, interaction designers, ethnographers and roboticists. The goal of this augmentation or robotization of the object is to improve the 


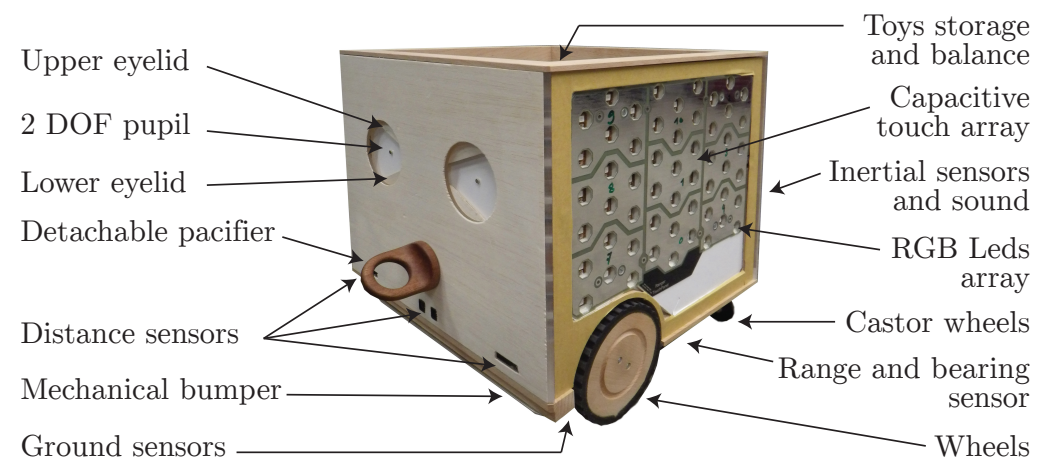

Fig. 2: The ranger robot main components and their locations. The eyeglasses have been removed for the purpose of the illustration.

functionalities (using competences in robotics and mechatronics) by building on the top of an existing interaction and affordance (using competences in interaction design) and create a new form of robot that can be accepted in an existing ecosystem (using competences in ethnography).

The resulting ranger robot has a body based on the original wooden box but is equipped with wheels, mechanical eyes, inertial sensors, distance sensors, ground sensors, a bumper, an inside balance, capacitive external touch sensors, LED panels behind the wooden surface, sound, eyeglasses, a detachable pacifier and a range-and-bearing $(\mathrm{R} \& \mathrm{~B})$ system to detect other rangers, the battery charger and a beacon to know where the play zone is (see figure 2 for most details and figure 1 for a global view). Its body is shaped to support a very specific interaction aimed at encouraging the children to tidy up their room. Instead of maximizing the robotics functionalities for this type of application, the optimization is made at a higher level, taking into account the capabilities of the children, the ecosystem and the possible interaction. Therefore, we made the choice to not equip the ranger with arms, for instance, because this adds complexity and cost that can be avoided using the right form of interaction. The new robject is supposed to remain helping and interacting with the children instead of becoming a tool that does a task on its own. Finally, by keeping a role close to the original storage box, the ranger increases its acceptance by the children and their parents.

The internal architecture of the robot is illustrated in figure 3 . The ranger has four processors. The main one is a full embedded computer running Linux and providing the main wireless connectivity, data storage and sound management. Three micro-controllers, connected using a CAN bus, manage the real-time part of the robot, including power, sensors and actuators. The ASEBA framework [20] is used to control the whole system and makes the link with ROS controllers. Slam and navigation are implemented using ROS. 
The shape of the ranger has been designed to keep a neutral wooden surface that can become a projection surface from inside. The 186 RGB LEDs that can project light on this surface can create a large set of animated patterns. The eyes are fully mechanical, without any screen, creating a coherent image of real device. The eyelids allow to give more expression and close the eyes when inactive. The eyeglasses of different colors allow a protection of the mechanics of the eyes and at the same time allow a distinction among the several rangers holding several types of toys, for instance. The pacifier is also made of wood but includes a magnet to be placed in the "mouth" of the rangers. For some experiments we add a RGDB Primesense sensor connected by USB to the Linux board and helping in navigation and obstacle detection.

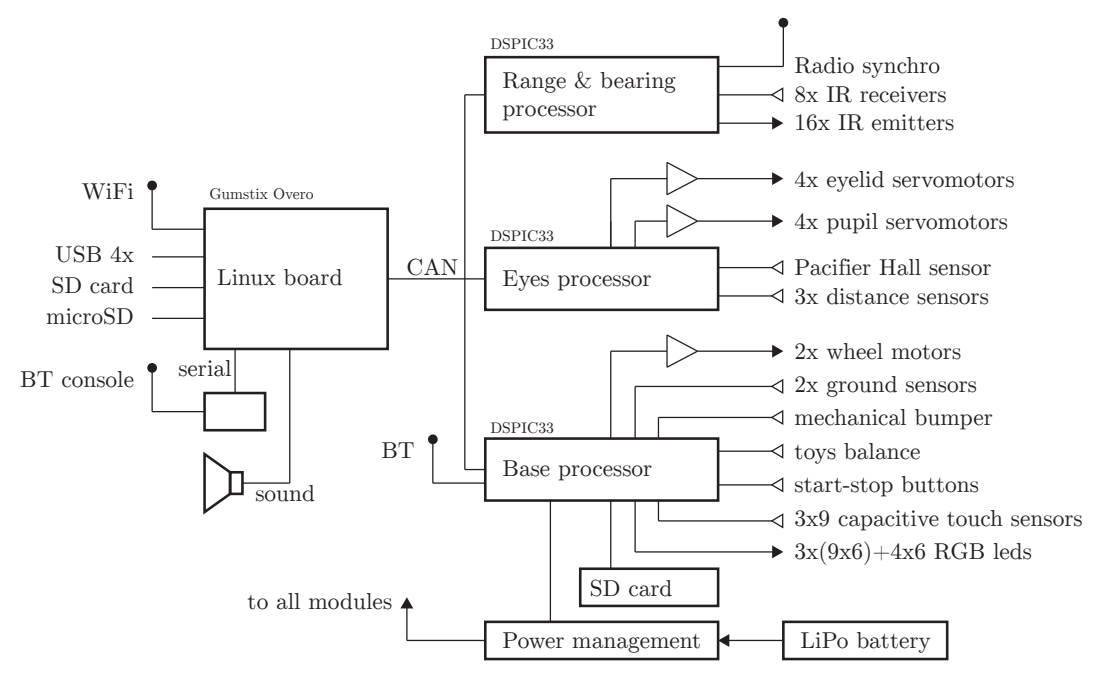

Fig. 3: The structure of the electronics of the ranger robot. Four processors manage the functionalities and can be expanded using the USB ports of the Linux board.

\subsection{Ranger use scenario}

The use scenario is based on a room equipped with a bed under which several rangers can be stored (see figure 1). Under the bed we placed, for each ranger, a recharging station equipped with a $\mathrm{R} \& \mathrm{~B}$ beacon. This is the home of the rangers where they sleep, with closed eyes, most of the time. Their behavior is defined by a state machine. Figure 4 shows a simplified version of the state machine controlling the ranger behavior.

The children have a controller object (called "collector") where they can place up to three pacifiers. This collector of pacifiers can be seen on the bottom, 

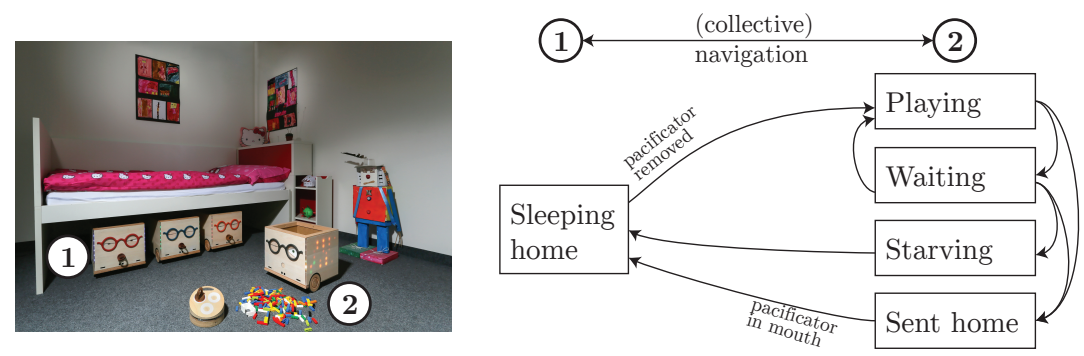

Fig. 4: The behavior of the ranger is based on two positions: under the bed (home) and on the playing site. At home the ranger can be charged and the playing site is marked by a beacon where the children place the pacifiers.

center, of figure 1. The collector is equipped with a beacon. To play with their toys, the children identify which ranger hold them. Each ranger has a different color of glasses. The child can wake up the right ranger by taking away the pacifier from its mouth. The child then puts the pacifiers in the collector placed where the children want to play. The ranger will wake up and navigate toward the collector beacon. Once the child wants to send back the ranger home, he can simply put again the pacifiers in its "mouth" and the ranger will go back home to sleep (and recharge). The ranger can also decide to go back home if it sees that the battery level is too low. A set of behaviors is used to keep the child engaged. It is motivated to tidy up and fill ranger by the robot showing signs of hunger and sadness if he not being "fed" - while displaying happiness as a rewarding behavior if toys are being placed inside ranger.

\section{$3.2 \quad$ Validation}

We tested the ranger concept in 14 families in a first short-term study [21]. One ranger prototype was placed in the room of the children while a researcher was discussing with the family in another room. The family was then asked to go in the room of the children and discover the ranger. The ranger was remotely controlled from a third room through a hidden camera, following a predefined scenario describing the behavior, following a Wizard-of-Oz methodology [22]. In half of the families we adopted a reactive behavior, with the ranger waiting for actions of the children before reacting. In the other half we adopted a proactive behavior, with the ranger proactively moving and looking for objects on the ground.

These tests have shown a very high acceptance, as illustrated by the detailed results presented in [21]. Moreover we observed that marginally significantly more toys were put and removed in the reactive compared to the proactive behavior. This demonstrates that the robot can achieve good performances even with a minimalist behavior. 


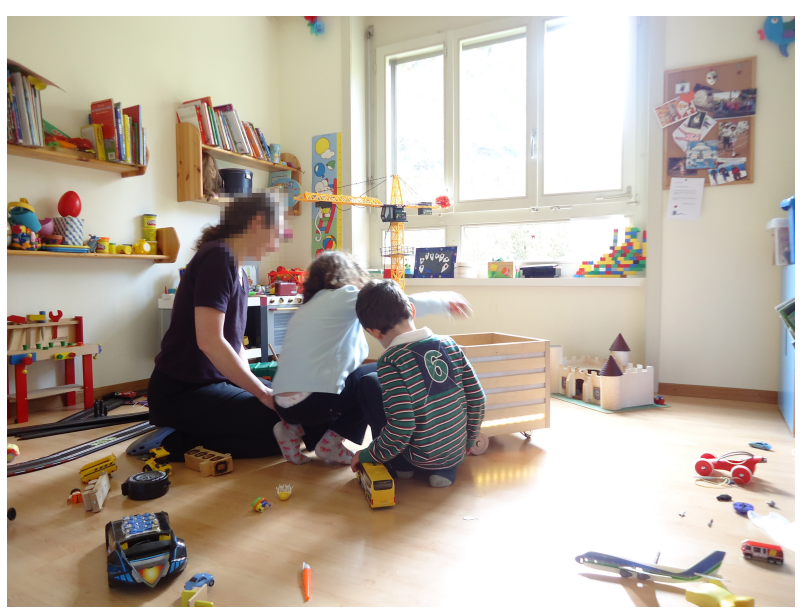

Fig. 5: An image taken during user tests in families.

The next step consists in making tests with users in a configuration similar to the one presented in figure 1 and for a long period, to see to what extent users engage in using the ranger after novelty effects have worn off.

\section{Conclusion}

We presented the concept and design of the ranger robot, a robotic storage box that can interact with children to motivate them to tidy-up their room. This concept is very similar to the one adopted by several successful industrial robotics systems that have shown the path of a smart integration of human and robotic activities, limiting the requirements for the robotic technology and improving performances thanks to a good human-robot cooperation. Following this design approach, we can design robots that fit with the factors identified by Bauwens et al. [5]: practical utility, integration in the ecosystem and economic utility. The validation in families has shown that ranger achieves high acceptance rates and a good utility in short-time experiments. In parallel, this test shows that good performances can be achieved with a minimal behavior, helping to reach the economic utility. These results are achieved because of the embodiment into a daily-life object, ensuring an optimal affordance and integration into the home ecosystem. This example illustrates the potential of the robjects concept but also shows that this concept requires an holistic approach that integrates interaction design, robotics and mechatronics design.

\section{Acknowledgment}

This work was supported by the Swiss National Center of Competence in Research "Robotics" 


\section{References}

1. B. Gates, "A Robot in Every Home," Scientific American, vol. 296, pp. 58-65, 2007.

2. Eurobarometer, "Public Attitudes Towards Robots," Tech. Rep. March, 2012.

3. C. Pantofaru, L. Takayama, T. Foote, and B. Soto, "Exploring the role of robots in home organization," Proceedings of the seventh annual ACM/IEEE international conference on Human-Robot Interaction - HRI '12, p. 327, 2012.

4. G. Bell, M. Blythe, and P. Sengers, "Making by making strange: Defamiliarization and the design of domestic technologies," ACM Trans. Comput.-Hum. Interact., vol. 12, pp. 149-173, June 2005.

5. V. Bauwens and J. Fink, "Will your household adopt your new robot?," Interactions, vol. 19, p. 60, Mar. 2012.

6. I. Duque, K. Dautenhahn, K. L. Koay, I. Willcock, and B. Christianson, "Knowledge-driven user activity recognition for a smart house. development and validation of a generic and low-cost, resource-efficient system," in ACHI 2013, The Sixth International Conference on Advances in Computer-Human Interactions, pp. 141-146, 2013.

7. F. Cavallo, M. Aquilano, M. Bonaccorsi, R. Limosani, A. Manzi, M. C. Carrozza, and P. Dario, "On the design, development and experimentation of the ASTRO assistive robot integrated in smart environments," Robotics and Automation (ICRA), 2013 IEEE International Conference on and ..., vol. 2, pp. 4310-4315, May 2013.

8. M. Aquilano, M. C. Carrozza, and P. Dario, "Robot-Era project (FP7-ICT2011.5.4): from the end-users perspective to robotics. Preliminary findings.," in Proceedings of the AAL - Ambient Assisted Living Forum 2012, (Eindhoven, The Netherlands), 2012.

9. S. Coradeschi, A. Cesta, G. Cortellessa, L. Coraci, J. Gonzalez, L. Karlsson, F. Furfari, A. Loutfi, A. Orlandini, F. Palumbo, F. Pecora, S. von Rump, A. Štimec, J. Ullberg, and B. Östlund, "Giraffplus: Combining social interaction and long term monitoring for promoting independent living," in In Proceedings of Human System Interaction (HSI) 2013, pp. 578-585, Ieee, June 2013.

10. F. Amirabdollahian, R. op den Akker, S. Bedaf, R. Bormann, H. Draper, V. Evers, G. Gelderblom, C. Gutierrez Ruiz, D. Hewson, N. Hu, I. Iacono, K. Koay, B. Krose, P. Marti, H. Michel, H. Prevot-Huille, U. Reiser, J. Saunders, T. Sorell, and K. Dautenhahn, "Accompany: Acceptable robotics companions for ageing years - multidimensional aspects of human-system interactions," in Human System Interaction (HSI), 2013 The 6th International Conference on, pp. 570-577, June 2013.

11. M. Nani, P. Caleb-solly, S. Dogramadgi, C. Fear, and H. V. D. Heuvel, "MOBISERV : An Integrated Intelligent Home Environment for the Provision of Health , Nutrition and Mobility Services to the Elderly," in 4th Companion Robotics Workshop in Brussels, 2010.

12. H. M. Gross, C. Schroeter, S. Mueller, M. Volkhardt, E. Einhorn, A. Bley, C. Martin, T. Langner, and M. Merten, "Progress in developing a socially assistive mobile home robot companion for the elderly with mild cognitive impairment," in IEEE International Conference on Intelligent Robots and Systems, pp. 2430-2437, 2011.

13. D. Fischinger, P. Einramhof, W. Wohlkinger, K. Papoutsakis, P. Mayer, P. Panek, T. Koertner, S. Hofmann, A. Argyros, M. Vincze, et al., "Hobbit-the mutual care robot," in Workshop-Proc. of ASROB, 2013. 
14. D. Bacciu, P. Barsocchi, S. Chessa, C. Gallicchio, and A. Micheli, "An experimental characterization of reservoir computing in ambient assisted living applications," Neural Computing and Applications, vol. 24, pp. 1451-1464, Mar. 2013.

15. J. Sequeira, P. Lima, A. Saffiotti, V. Gonzalez-Pacheco, and M. Salichs, "MOnarCH: Multi-robot cognitive systems operating in hospitals," in ICRA 2013 Workshop on Many Robot Systems, 2013.

16. H. Lehmann, M. Walters, A. Dumitriu, A. May, K. Koay, J. Saez-Pons, D. Syrdal, L. Wood, J. Saunders, N. Burke, I. Duque-Garcia, B. Christianson, and K. Dautenhahn, "Artists as hri pioneers: A creative approach to developing novel interactions for living with robots," in Social Robotics (G. Herrmann, M. Pearson, A. Lenz, P. Bremner, A. Spiers, and U. Leonards, eds.), vol. 8239 of Lecture Notes in Computer Science, pp. 402-411, Springer International Publishing, 2013.

17. P. Wurman, R. D'Andrea, and M. Mountz, "Coordinating hundreds of cooperative, autonomous vehicles in warehouses," AI Magazine, vol. 29, no. 1, pp. 9-20, 2008.

18. C. Fitzgerald and D. Ed, "Developing Baxter," in Technologies for Practical Robot Applications (TePRA), 2013 IEEE International Conference on, pp. 1-6, 2013.

19. L. Takayama, W. Ju, and C. Nass, "Beyond dirty, dangerous and dull: what everyday people think robots should do," Proceedings of the 3rd ACM/IEEE international conference on Human robot interaction, pp. 25-32, 2008.

20. S. Magnenat, P. Rtornaz, M. Bonani, V. Longchamp, and F. Mondada, "ASEBA: A Modular Architecture for Event-Based Control of Complex Robots," IEEE/ASME Transactions on Mechatronics, vol. 16, no. 2, pp. 321-329, 2011.

21. J. Fink, S. Lemaignan, P. Dillenbourg, P. Rétornaz, F. Vaussard, A. Berthoud, F. Mondada, F. Wille, and K. Franinovic, "Which Robot Behavior Can Motivate Children to Tidy up Their Toys ? Design and Evaluation of Ranger ," in Proceedings of the 2014 ACM/IEEE international conference on Human-robot interaction, pp. 439-446, 2014.

22. P. Green and L. Wei-Haas, "The rapid development of user interfaces: Experience with the wizard of oz method," in Proceedings of the Human Factors and Ergonomics Society Annual Meeting, vol. 29, pp. 470-474, SAGE Publications, 1985. 